REGULATORY WATCH

\section{Outcomes of early health technology assessment dialogues in medicinal product development}

The development of medicinal products has been shifting towards a value-driven model. However, although companies need to engage with health technology assessment (HTA) bodies to inform effective strategies to gain market access for their new medicinal products, HTA scientific advice (SA) procedures are not yet routine steps in the clinical development of such products.

In 2009, the UK's National Institute for Health and Care Excellence (NICE) launched its own HTA-only single-country SA procedure, known as NICE SA. NICE also participates in multi-country HTA-only joint SA procedures and in single- and multi-country parallel regulatory and HTA procedures with the UK's Medicines and Healthcare products Regulatory Agency (MHRA) and the European Medicines Agency (EMA). Here, we report the experience of NICE in giving HTA SA to companies between 2009 and 2015 .
In this time period, NICE SA completed 166 SA procedures involving medicinal products. These procedures included 105 providing NICE SA (63\%), 45 EMA-HTA parallel advice projects (27\%), 9 SEED (Shaping European Early Dialogues for health technologies) projects (5.5\%), 4 MHRA-HTA procedures $(2.5 \%)$ and 3 EUNetHTA procedures (2\%). NICE was involved in $75 \%$ of the EMA-HTA SA procedures (48 out of a total of 63 completed procedures). The last 2 years saw an increase in the number of SA procedures involving other HTA bodies of the European Union and the EMA.

FIGURE 1a presents the outcomes known to date for the 166 products for which NICE SA has issued advice. In total, $16(10 \%)$ of the products to date have received a marketing authorization in the European Union and one has received a positive opinion from the Committee for Medicinal Products for Human Use (CHMP). So far, clinical development has

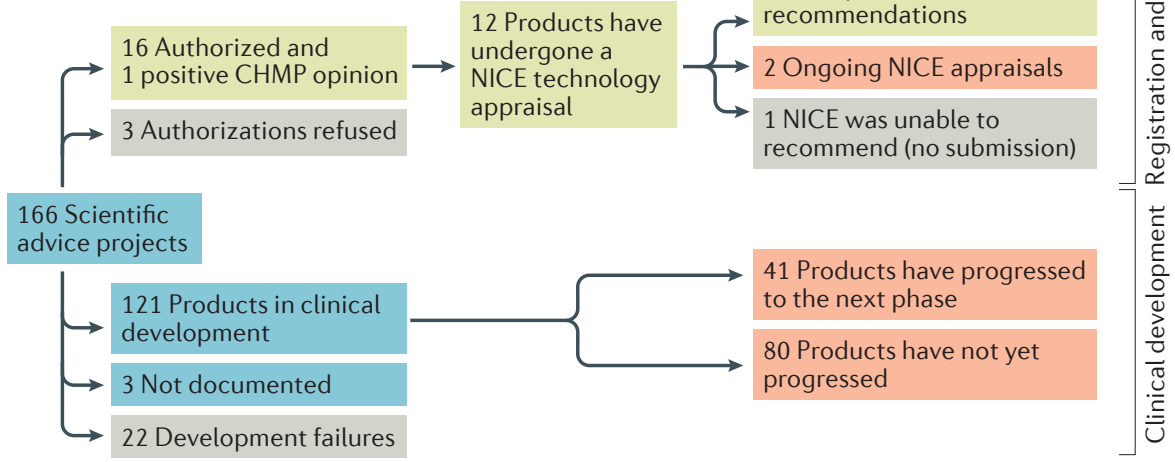

b

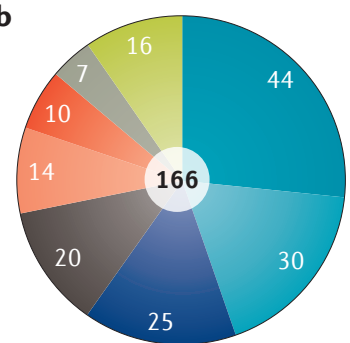

C

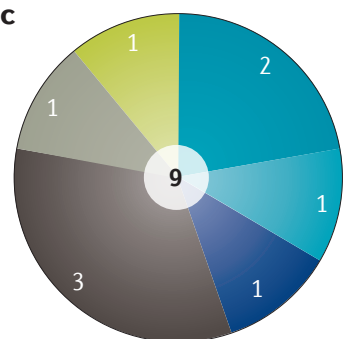

Antineoplastic and immunomodulating agents Nervous system Cardiovascular system Alimentary tract and metabolism Respiratory system Anti-infectives for systemic use Other Musculoskeletal system
Figure 1 | Outcomes and data on NICE scientific advice. a | Outcomes for 166 medicinal products for which the National Institute for Health and Care Excellence (NICE) provided scientific advice between 2009 and 2015. b,c|Therapeutic areas of the 166 products and 9 products that subsequently received a positive recommendation from a NICE technology appraisal committee. CHMP, Committee for Medicinal Products for Human Use; HTA, health technology assessment. been terminated for 22 products (13\%), mostly for efficacy reasons. This number is likely to increase as most development programmes are ongoing. Marketing authorization was refused in three instances.

NICE SA procedures are still largely dominated by medicinal products developed by the largest pharmaceutical companies; of the 166 SA procedures, 156 (94\%) involved large pharmaceutical companies and 10 (6\%) projects involved small and medium-sized enterprises (SMEs). However, uptake of NICE SA from SMEs is slowly starting to increase, with 4 of the total of 10 SA procedures involving SMEs being conducted in 2014 and 6 in 2015. Notably, 6 of these procedures were for advanced therapies, an area in which SMEs are heavily involved and for which early dialogue could be particularly valuable, given the high novelty of the products and their development paths. Of the medicinal products for which SA was sought, 73\% (122) were innovative therapies, which legally would fall under the mandatory scope of the European centralized procedure.

The therapeutic areas of the 166 products that have received SA and the 9 approved products that subsequently received a positive recommendation by a NICE technology appraisal committee are shown in FIG. 1 b. The committee was unable to issue a recommendation for one product (in the absence of submission by the manufacturer), and the technology appraisal was still ongoing in two instances.

It is likely that HTA SA will continue to evolve in order to facilitate increasing collaborations among HTA bodies and between HTA bodies and regulatory agencies, and to complement new initiatives, such as the priority medicines initiative (PRIME). Our experience also shows that HTA bodies can learn from engaging in SA activities, such as gaining insight into novel trial designs and considering schemes for managed access. This prompts timely commissioning of methodological work and helps manage the expectations of decision-makers.

François Maignen is at The Office of Health Economics Southside, 7th Floor, 105 Victoria Street, London SWIE 6OT, UK.

Leeza Osipenko, Pilar Pinilla-Dominguez and Emily Crowe are at the Centre for Health Technology

Evaluation, National Institute for Health and Care Excellence, 10 Spring Gardens, London SW1A 2BU, UK.

Correspondence to F.M. FMaignen@ohe.org doi:10.1038/nrd.2016.286 Published online 2 Feb 2017

Competing interests statement

The authors declare no competing interests. 